\title{
Untersuchung und MR-Morphologie des ulnokarpalen Handgelenkschmerzes
}

\section{Übersicht}

Einleitung

Diagnostik

\section{Zusammenfassung}

Die Untersuchung des ulnokarpalen Anteils des Handgelenks ist ausgesprochen schwierig.

Diese Arbeit soll eine sinnvolle Zusammenstellung hilfreicher Tests abbilden, die im Ambulanz- alltag rasch durchgeführt werden können und zur exakten Diagnosefindung beitragen. Die Bedeutung einer hochwertigen MRT-Untersuchung wird durch die Darstellung der einzelnen Krankheitsbilder betont. In Tab. 2 am Ende des Beitrags sind nochmals alle beschriebenen Tests aufgelistet und - da durch einzelne Test mehrere Pathologien aufgedeckt werden können - die entsprechenden möglichen Diagnosen aufgeführt.

\section{Einleitung}

Die Untersuchung des Handgelenks bei ulnarseitigem Schmerz stellt im klinischen Alltag erfahrungsgemäß eine große Herausforderung dar. Folgende Gründe können dafür benannt werden:

- Anatomie: Das komplexe Zusammenspiel von intraund extrakapsulären Strukturen setzt profunde anatomische und biomechanische Kenntnisse voraus. Exemplarisch seien genannt:

- ulnokarpaler Komplex (TFCC)

- Sehne des M. extensor und flexor carpi ulnaris (ECU und FCU)

- distales Radioulnargelenk (DRUG)

- Schmerzqualität: In vielen Fällen fällt es dem Patienten schwer, den Schmerz genau zu lokalisieren oder zu provozieren. „Gerade heute tut die Hand nicht weh“ ist ein oft gehörter Satz, und man ist gut beraten, den Patienten nicht mit der Empfehlung zu entlassen, er solle wiederkommen, wenn der Schmerz bestehe. Es ist durch die räumliche Nähe der Strukturen und Schmerzausstrahlung oft schwierig, wenn nicht unmöglich, eine punktgenaue Lokalisation anzugeben. Hier ist der Untersucher gefordert, durch möglichst exaktes Palpieren den Schmerzpunkt herauszufinden. Hilfreich ist die passive Mobilisation einzelner Strukturen, unterstützt von ausgewählten Provokationstests.

- Bildgebung: Die überwiegende Anzahl von Pathologien, die für ulnokarpalen Schmerz verantwortlich sind, kann im konventionellen Röntgen nicht darge- stellt werden. Auch die Computertomografie ist meist (Ausnahme: Frakturen der Karpalknochen, speziell Frakturen oder Pseudarthrosen des Hamulus) nicht zielführend. Die Ultraschalldiagnostik mag in den Händen erfahrener Spezialisten durchaus hilfreich sein, im klinischen Alltag spielt sie eine eher untergeordnete Rolle. Die Arthroskopie wird oft als „Goldstandard“ gepriesen. Abgesehen davon, dass das Verfahren invasiv und teuer ist und große Erfahrung voraussetzt, haben Shin u. Mitarb. [1] nachgewiesen, dass bei $25 \%$ der Patienten mit ulnokarpalem Handgelenkschmerz eine extraartikuläre Ursache verantwortlich ist. Diese kann naturgemäß arthroskopisch weder diagnostiziert noch therapiert werden. Die Magnetresonanztomografie (MRT) zeigt im Gegensatz dazu alle Strukturen des Ulnokarpalgelenks und kann wertvolle Hilfe leisten.
Abkürzungen
DRUG distales Radioulnargelenk
ECU Extensor carpi ulnaris
EPL Extensor pollicis longus
FCU Flexor carpi ulnaris
MRT Magnetresonanztomografie
TFCC triangular fibrocartilage complex
(ulnokarpaler Komplex)
LT lunotriquetral
PD proton density
TILT triquetral impingement ligament tear 


\section{Tabelle 1}

Häufige Ursachen von ulnokarpalem Schmerz.

\begin{tabular}{|l|l|}
\hline Lokalisation & Krankheitsbild \\
\hline TFCC & $\begin{array}{l}\text { Ruptur, Synovialitis, Ulna Impaction } \\
\text { Syndrome }\end{array}$ \\
\hline DRUG & Synovialitis, Arthrose \\
\hline ECU-Sehne & Synovialitis, (Sub-)Luxation, (Partial-)Ruptur \\
\hline Verletzung des Lig. lunotriquetrum & (Partial-)Ruptur \\
\hline Triquetral Impingement Ligament & Abriss Lig. radiolunotriquetrum dorsale \\
\hline Tear (TILT) & \\
\hline Os pisiforme & pisotriquetrale Arthrose, Subluxation \\
\hline Hamulus ossis hamati & Fraktur, Pseudarthrose \\
\hline Triquetrum, Hamatum & Fraktur, Bone Bruise, Chondromalazie \\
\hline FCU-Sehne & Synovialitis, (Partial-)Ruptur \\
\hline
\end{tabular}

Im heutigen Ambulanzalltag ist Zeit so kostbar wie nie. Ziel dieser Arbeit ist deshalb, dem Untersucher bewährte Techniken vorzustellen, die einerseits leicht erlernbar sind, andererseits möglichst rasch zu einer Diagnosefindung beitragen. Es ist nicht beabsichtigt, den Leser mit einer möglichst vollständigen Aufzählung der in der Literatur beschriebenen Tests zu konfrontieren. Viele unterscheiden sich nur marginal, andere haben sich in der Praxis als wenig hilfreich erwiesen.

In Tab. 1 sind Krankheitsbilder aufgeführt, deren Ursache entweder intra- oder extrakapsulär liegt und für mehr als 95\% des Symptoms „ulnokarpaler Schmerz“ verantwortlich ist. Wir diskutieren anhand dieser Krankheitsbilder die effektivsten Untersuchungsmethoden. Zwangsläufig ergeben sich bei einzelnen Tests Überschneidungen; am Ende (Tab. 2) werden wir deshalb nochmals tabellarisch die sinnvollen Untersuchungen mit den entsprechenden Krankheitsbildern verknüpfen.

In vorhergehenden Arbeiten wurden zur Verdeutlichung der topografischen Anatomie Röntgenbilder den Untersuchungen gegenübergestellt. Da wie oben beschrieben wesentliche Strukturen, die für ulnokarpalen Schmerz verantwortlich sind, im konventionellen Röntgen nicht dargestellt werden können, verwenden wir MRT-Aufnahmen. Die Bilder wurden mit einem 3-Tesla-MRT unter Verwendung einer Handspule in Submillimetertechnik gewonnen. Es ist das Verdienst der radiologischen Kollegen der Hessingpark-Clinic in Augsburg, für jede Fragestellung ein maßgefertigtes Untersuchungsprotokoll durch Festlegung der Sequenzen erstellt zu haben.

Nach unserem Verständnis kann eine optimale Diagnosefindung nur in Zusammenarbeit von Handchirurg und Radiologen erfolgen. Je präziser die Fragestellung von handchirurgischer Seite gestellt wird, umso besser kann die Bildgebung erfolgen.

\section{Diagnostik}

\section{Anamnese}

Schmerzen an der Ulnarseite des Handgelenks können durch eine akute Verletzung (Sturz, Verdrehtrauma) oder chronische Überlastung ausgelöst werden. Chronisch-entzündliche Prozesse lassen sich häufig anamnestisch eruieren. Der Patient muss befragt werden nach der Schmerzlokalisation und -dauer, nach Ruhe- und/oder Belastungsschmerz und möglichen Bewegungen, durch die der Schmerz provozierbar ist. Selbstredend sollen bisher durchgeführte Therapiemaßnahmen und eventuelle Arbeitsunfähigkeitszeiträume bekannt sein.

\section{Inspektion}

Bei der Inspektion richtet sich das Augenmerk auf verdächtige Schwellungen, Veränderungen des Hautkolorits, atypische Venenzeichnungen oder Fehlstellungen.

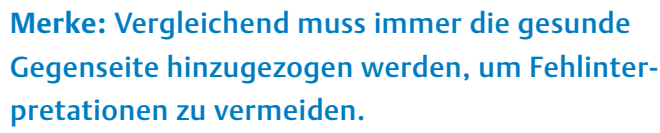

Garcia-Elias [2] hat ein nützliches Zeichen beschrieben, mit dessen Hilfe ein prominenter Ellenkopf den entscheidenden Hinweis auf eine multidirektionale Instabilität des DRUG liefert: Versucht der Patient, mit der flachen Hand Druck auf die Tischplatte auszuüben, wandert der Ellenkopf nach palmar und wirft auf der Streckseite des Handgelenks ein Grübchen auf („dimple sign“). 


\section{Prüfung der Handgelenkbeweglichkeit}

Watson u. Weinzweig [3] stellen generell fest: „Ist eine vollständige Pronation und Supination im Unterarm möglich, kann eine Verletzung des TFCC oder des DRUG ausgeschlossen werden. “ Ist dagegen die passive Flexion des Handgelenks eingeschränkt, liegt eine karpale Pathologie vor.

Die klinische Untersuchung sollte zunächst mit der Dokumentation des passiven und aktiven Bewegungsumfangs an beiden Handgelenken beginnen. Die Beurteilung der Messungen erfolgt weniger am Absolutwert der Beweglichkeit, sondern in Relation zum kontralateralen, nicht betroffenen Handgelenk.

Der Untersucher sitzt dem Patienten gegenüber. Die beiden Ellenbogen sind gebeugt und ruhen auf dem Untersuchungstisch. Flexion und Extension werden nach der Neutral-0-Methode bestimmt. Der Goniometer wird streckseitig angelegt. Die Messung der Ulnarund Radialduktion erfolgt in Pronationsstellung des Handgelenks; die Handinnenfläche ruht entspannt auf der Unterlage. Beurteilt wird der Winkel zwischen Unterarmlängsachse und der Achse des 3. Mittelhandknochens. Pro- und Supinationsfähigkeit werden unter Adduktion der Oberarme an den Brustkorb bei gleichzeitiger $90^{\circ}$-Flexion im Ellenbogengelenk geprüft. In der Neutralposition liegen Handrückenebene und Humerusachse parallel in der Sagittalebene, unabhängig von der Stellung des Schultergelenks [4].

\section{Hintergrund}

Als Normwerte der physiologischen Handgelenkbeweglichkeit gelten folgende Werte:

- Extension/Flexion: 60-0-60

- Ulnar-/Radialduktion: 35-0-20

- Pronation/Supination: $80-0-80^{\circ}$

Merke: Es muss betont werden, dass erhebliche individuelle Unterschiede bestehen. Eine Streckfähigkeit von $30^{\circ}$ ist keine pathologische Einschränkung, wenn die gesunde Gegenseite nicht vermehrt gestreckt werden kann. Umgekehrt ist gerade bei jungen Frauen eine Streckung und Beugung von bis zu $90^{\circ}$ durchaus möglich.

Von großer Bedeutung ist die Einschätzung der Grobkraft und Feinmotorik (Pinchgriff). Die Messung sollte mit geeichten Dynamometern und zusätzlich an der gesunden Gegenseite durchgeführt werden. Nicht sel- ten ergibt sich in der Sprechstunde das Problem, dass der Patient just zum Untersuchungszeitpunkt den Handgelenkschmerz nicht provozieren kann. Es ist dann oft hilfreich, den Patienten wiederholt den Dynamometer drücken zu lassen. Durch die vermehrte Belastung wird der Schmerz provoziert und ist besser lokalisierbar.

Nach den Untersuchungen von Shaaban u. Mitarb. [5] ist die Kraftübertragung zwischen Ulna und Lunatum in Pronation höher als in Supination. LaStayo u. Weiss [6] konnten zeigen, dass Patienten mit einem ulnokarpalen „impaction syndrome“ in Pronation deutlich weniger Grobkraft entwickeln als in Supination („gripping rotatory impaction test“, GRIT-Test).

\section{Praxistipp}

Wichtig ist, bei der Untersuchung auch auf Geräusche zu achten: So verursacht eine bei Pro- und Supination luxierende ECU-Sehne ein schmerzhaftes und deutlich hörbares Schnappen. Eine mediokarpale Instabilität löst bei der aktiven und passiven Ulnarduktion ein Klickgeräusch aus, bedingt durch das plötzliche Springen der proximalen karpalen Reihe aus der (pathologischen) Flexion in die Extension.

\section{Palpation mithilfe der topografischen Anatomie}

Bestimmte Landmarken erleichtern dem Untersucher die Orientierung und sind nahezu immer zu finden, auch bei sehr adipösen Patienten oder starker, traumabedingter Schwellung.

Die Untersuchung beginnt von dorsal in Pronationsstellung des Handgelenks (Abb. 1). Die Hand ruht auf dem Untersuchungstisch, der Patient ist entspannt und flektiert den Ellenbogen im rechten Winkel. In Verlängerung des 2. Interdigitalraums ist in Höhe der Handgelenkstreckfurche das Tuberculum dorsale lokalisiert. Dieser markante Knochenpunkt dient als Hypomochlion für die Sehne des M. extensor pollicis longus (EPL). Etwa $1 \mathrm{~cm}$ distal davon liegt der radiokarpale Gelenkspalt. Etwa $2 \mathrm{~cm}$ medial des Tuberculum dorsale liegt der Ulnakopf. Rund 2/3 der Artikulationsfläche des Kopfes sind palpabel. Ulnarseitig ist als Endausläufer des Ellenkopfs der Processus styloideus ulnae tastbar. In Pronation des Handgelenks ist er weit palmar gelegen, mit zunehmender Supination nimmt er eine mehr dorsale Lage ein. Alternierende Supination und Pronation erleichtern das Auffinden des Prozessus. Distal des 
Abb. 1 a Topografie der Hand von dorsal. b MRTNormbefund des Handgelenks (koronal PD).
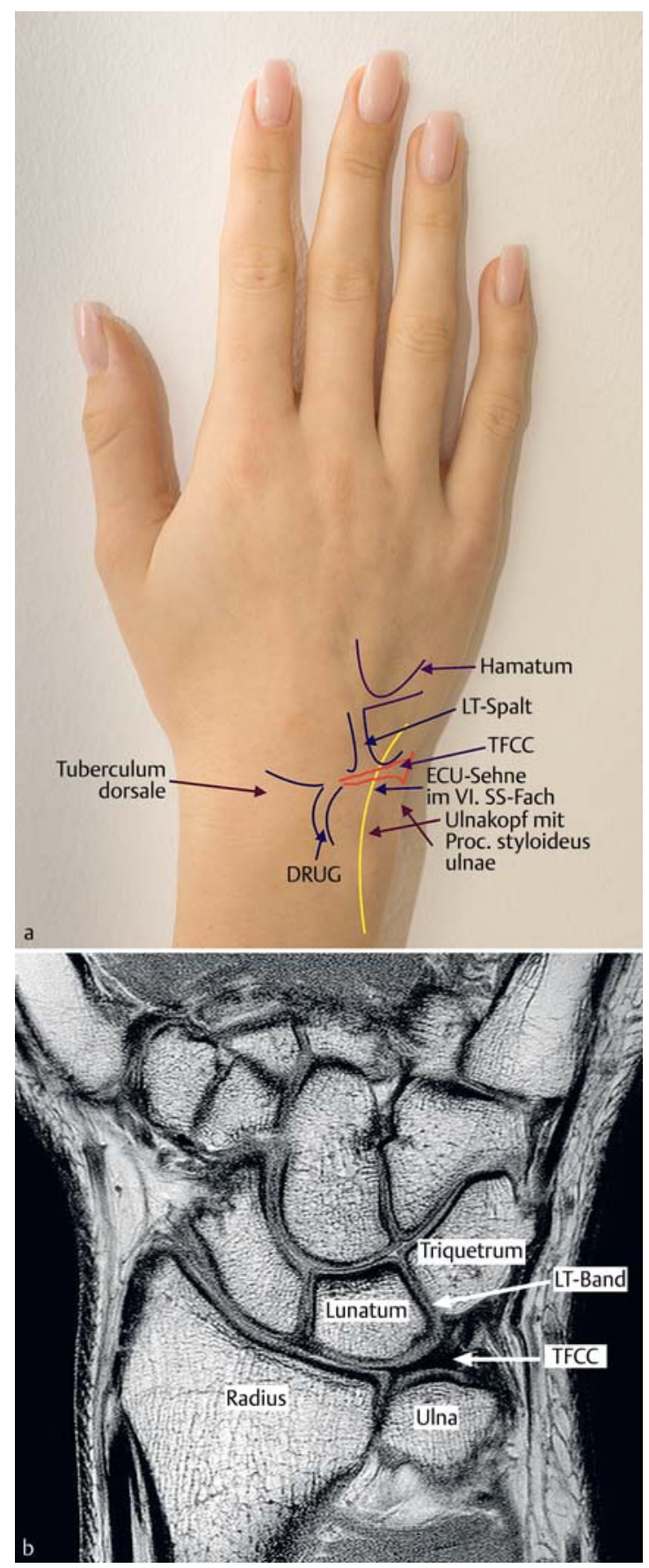

Prozessus liegt als wichtiger Stabilisator des distalen Radioulnargelenks der TFCC. Am besten lässt sich durch leichte Flexion und Radialduktion des Handgelenks die unmittelbar an den Ulnakopf angrenzende dorsale Kante des Triquetrums ertasten. Radial des Triquetrums liegt das Lig. lunotriquetrum im LT-Spalt, distal des Triquetrums unmittelbar angrenzend das Mediokarpalgelenk und das Hamatum.
Die weitere Untersuchung dorsaler Strukturen erfolgt nun in Supinationsstellung des Handgelenks. Der Patient stellt den Ellenbogen auf den Untersuchungstisch und wird gebeten, in seine Handfläche zu sehen. In dieser Stellung ist die ECU-Sehne sehr oberflächlich und lässt sich leicht im 6 . Strecksehnenfach palpieren. Direkt palmar der Sehne, eingegrenzt durch Ulna und Triquetrum, liegt die „foveal area“. Folgende Strukturen sind hier zu finden:

- R. dorsalis n. ulnaris

- Recessus praestyloideus

- ulnobasale Anheftung des TFCC

- Meniscus homologue

Entsprechend den Untersuchungen von Tay u. Mitarb. [7] wird Druckschmerz in dieser eng umschriebenen Region als positives „fovea sign“ beschrieben (Abb.2).

Bei der Untersuchung von palmar (Abb.3) liegt etwas distal der Raszetta oberflächennah das Os pisiforme. Etwa $2 \mathrm{~cm}$ distal und $2 \mathrm{~cm}$ radial davon ist in der Tiefe der Hamulus ossis hamati zu palpieren. Beginnend vom proximalen Rand des Os pisiforme erstreckt sich Richtung distaler Unterarm die FCU-Sehne. Der geübte Untersucher hat in wenigen Augenblicken diese Orientierungspunkte identifiziert und kann mit ihrer Hilfe die weiteren Untersuchungsschritte durchführen.

\section{Untersuchungstechnik: TFCC}

Merke: Die häufigste Ursache von ulnokarpalem

Handgelenkschmerz ist eine Verletzung des TFCC.

Eine exzellente Darstellung der komplexen Anatomie ist bei Nakamura [8] zu finden. Zusammenfassend sollen hier die einzelnen Anteile des TFCC erfasst werden:

- Triangular Fibrocartilage (entspricht dem Discus articularis)

- Meniscus homologue

- dorsales und palmares radioulnares Band

- Lig. ulnolunatum

- Lig. ulnotriquetrum

- Sehnenscheide des M. extensor carpi ulnaris

- ulnare Gelenkkapsel

Da die Strukturen direkt ineinander übergehen, müssen mehrere Untersuchungstechniken eingesetzt werden, um eine möglichst exakte Beschreibung der Läsion zu erhalten. 


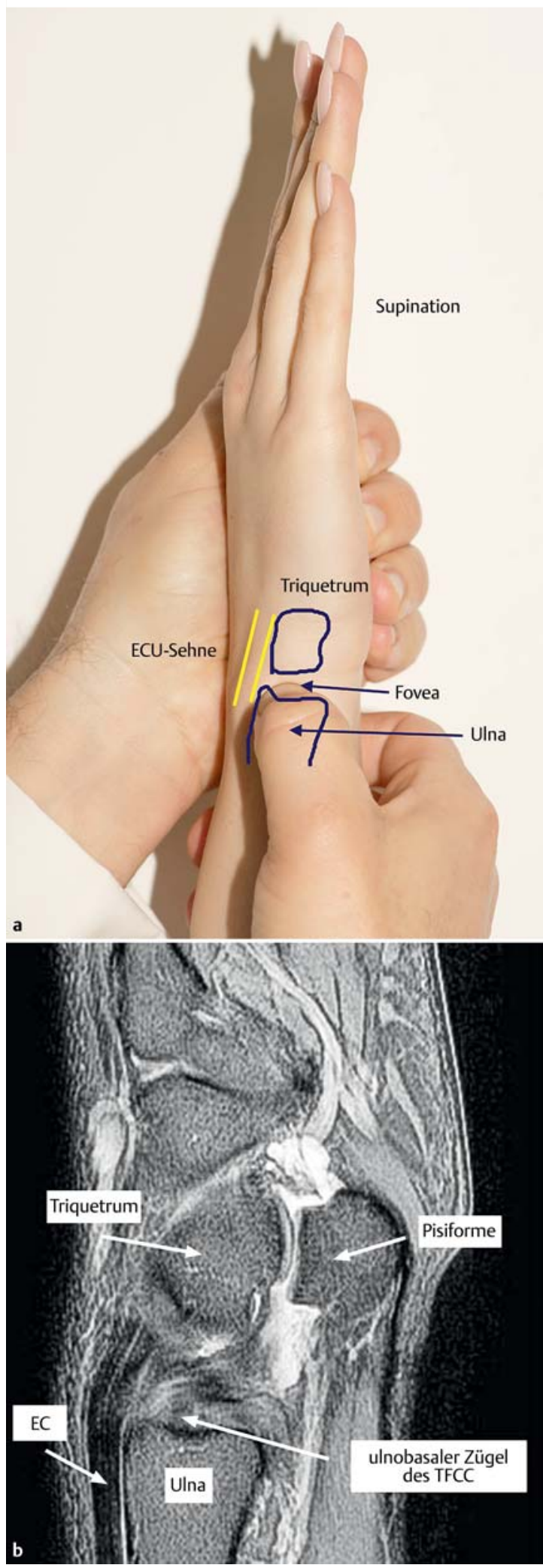

Abb.2 a Palpation des Handgelenks: „fovea sign“. b MRT: Fovea ulnae (sagittal, PD fettgesättigt).

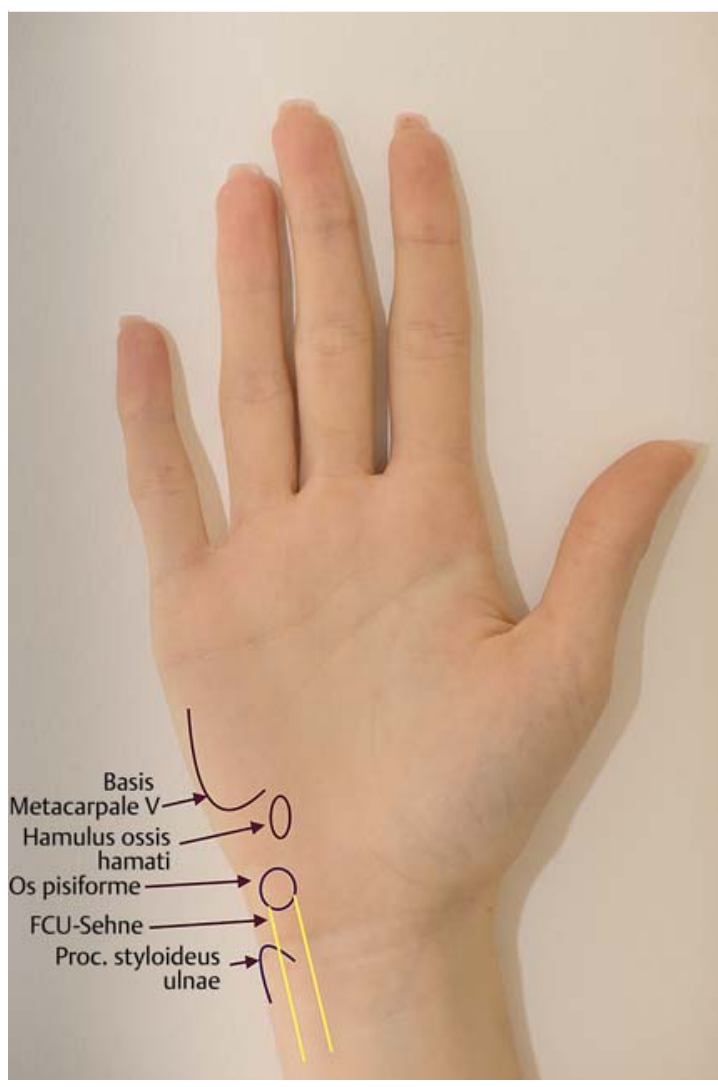

Abb.3 Topografie der Hand von palmar.

\section{- Fovea Sign}

Zunächst wird die Fovea ulnae aufgesucht. Der Patient stellt den Ellenbogen auf den Untersuchungstisch und betrachtet seine Handinnenfläche. Der Untersucher palpiert von ulnar kommend die Fovea im Dreieck zwischen ECU-Sehne, Ulna und Triquetrum (Abb.2). Nach Tay u. Mitarb. [7] gibt Schmerz über der Fovea einen Hinweis auf eine mögliche Verletzung des Lig. ulnotriquetrum oder einen Abriss der fovealen (tiefen) Insertion des TFCC. Die Sensitivität wird mit 95\%, die Spezifität mit $86 \%$ angegeben.

\section{- TFCC Shear Test}

Beschrieben von Berger u. Dobyns [9], beruht dieser Test auf einer passiven Mobilisation des TFCC. Im Vergleich zur Voruntersuchung dreht der Patient das Handgelenk in die Neutralstellung. Die Finger werden vom Untersucher locker gefasst; mit dem Zeigefinger wird Druck in dorsaler Richtung auf das Os pisiforme, mit dem Daumen gleichzeitig Druck in palmarer Richtung auf den Ulnakopf ausgelöst (Abb.4). Nach den Untersuchungen von LaStayo u. Howell [10] ist der Test bei $66 \%$ aller TFCC-Verletzungen positiv. 
Abb. 4 TFCC Shear Test.

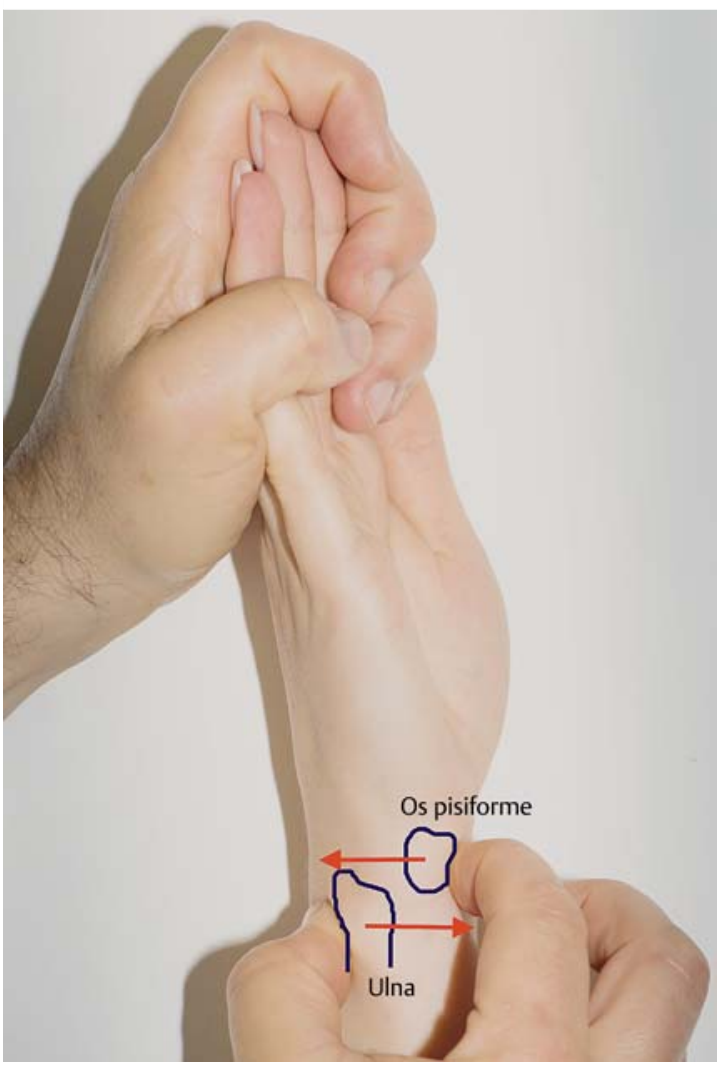

\section{Ulnocarpal Stress Test}

Dieser Test wurde als Provokationstest 1997 erstmalig von Nakamura u. Mitarb. [11] beschrieben. Das Handgelenk steht in Pronation. Eine Hand des Untersuchers stabilisiert den Unterarm, die andere Hand umfasst die Metakarpalia. Der TFCC wird nun durch 3 Kraftvektoren unter Stress gesetzt: axiale Kraft, Ulnarduktion und Rotation von Pro- zur Supination (Abb. 5). Bei einer Verletzung des TFCC wird heftiger Schmerz, manchmal begleitet von einem Schnappen, ausgelöst.

\section{Untersuchungstechnik: distales Radioulnargelenk}

\section{Dimple Sign}

Wie bereits oben beschrieben, ist es ein Hinweis auf eine multidirektionale Instabilität. Der Patient drückt mit der flachen Hand auf die Tischplatte. Der Ellenkopf rutscht weit nach palmar und wirft auf der Streckseite des Handgelenks ein Grübchen auf.

\section{Piano Key Test}

Ein wertvoller und einfach durchführbarer Test zum Nachweis einer Instabilität. In Modifikation zur Erstbeschreibung durch Beckenbaugh u. Mitarb. [12] lassen
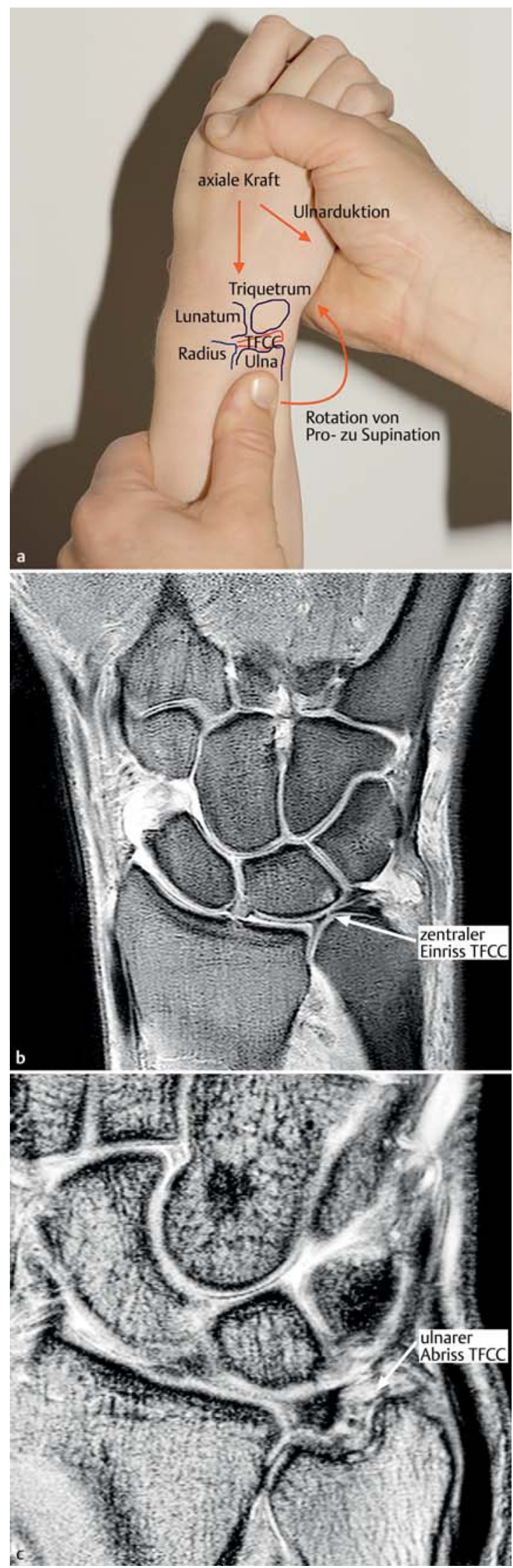

Abb. 5 a Ulnocarpal Stress Test. b MRT: zentrale Perforation TFCC (koronal PD). c MRT: ulnarer Abriss TFCC (koronal PD fettgesättigt). 
wir die flache Hand des Patienten gegen die Hand des Untersuchers drücken. So sind reflektorisch schmerzbedingte Anspannungen sofort erkennbar (in der Originalbeschreibung wird der Druck gegen eine Unterlage ausgeübt). Der Zeigefinger des Untersuchers versucht, den pronierten Ellenkopf nach palmar zu schieben (Abb.6); endet der Druck, springt der Ellenkopf wie eine Klaviertaste nach dorsal.

\section{DRUG Instability Test}

Bereits 1979 von Morrissy u. Mitarb. [13] beschrieben, muss dieser Test in Pronation, Neutralstellung und Supination durchgeführt werden. Der Radius wird mit einer Hand umfasst, der Ulnakopf mit der anderen Hand in dorsopalmarer Richtung verschoben (Abb.7). In Neutralstellung ist ein Bewegungsspiel von einigen Millimetern physiologisch, in Supination sollte dagegen eine feste Verriegelung vorliegen.

Merke: Bei diesem Test ist besonders wichtig, auch die gesunde Gegenseite zu prüfen, um Fehlbeurteilungen zu vermeiden.

\section{- Radioulnar Compression Test}

Das Handgelenk steht in Pronation. Nach Cooney u. Mitarb. [14] wird der Ellenkopf gegen den fixierten Radius in transversaler Richtung gepresst; gleichzeitig führt die andere Hand des Untersuchers eine Rotationsbewegung bis zur maximalen Supination durch (Abb.8). Schmerzhaftes Reiben im Gelenk ist ein Hinweis auf arthrotische Veränderungen.

\section{Untersuchungtechnik: Extensor-carpi- ulnaris-Sehne}

\section{- ECU Synergy Test}

Ruland u. Hogan [15] entdeckten, dass eine isometrische Kontraktion der Mm. abductor pollicis longus et brevis zu einer reflektorischen Anspannung der ECUSehne führt, um die Balance der radial- und ulnarseitigen Kräfte zu gewährleisten. Das Handgelenk steht in Supination, Daumen und Zeigefinger des Patienten werden vom Untersucher adduziert gehalten. Der Patient muss dann den Daumen gegen Widerstand abduzieren (Abb.9). Liegt eine Synovitis der ECU-Sehne vor, verspürt der Patient deutlichen Schmerz.

Die klassische Untersuchung (Handgelenk in Extension, aktive Ulnarduktion gegen Widerstand) verursacht dagegen Stress auf das Lig. lunotriquetrum und den TFCC und ermöglicht keine Unterscheidung zur extrakapsulären Pathologie der ECU-Sehne.

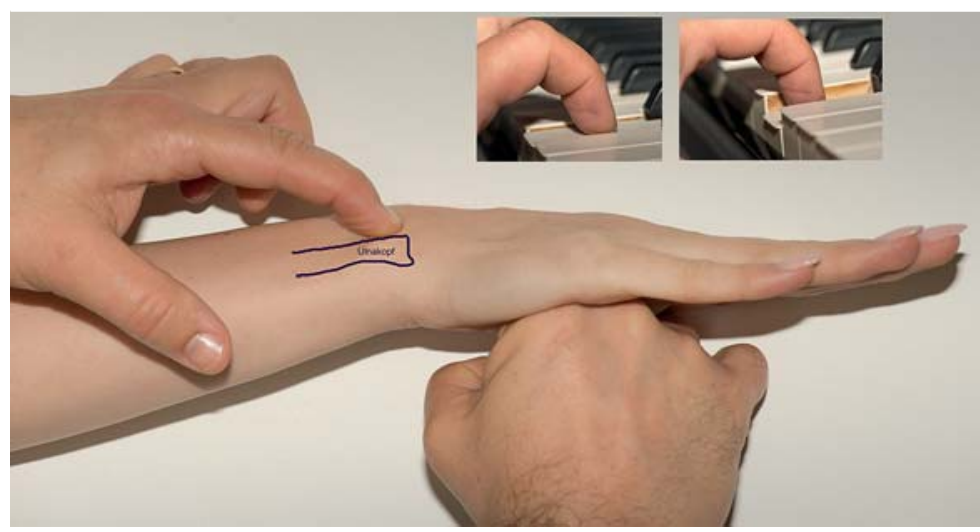

Abb. 6 Piano Key Test.
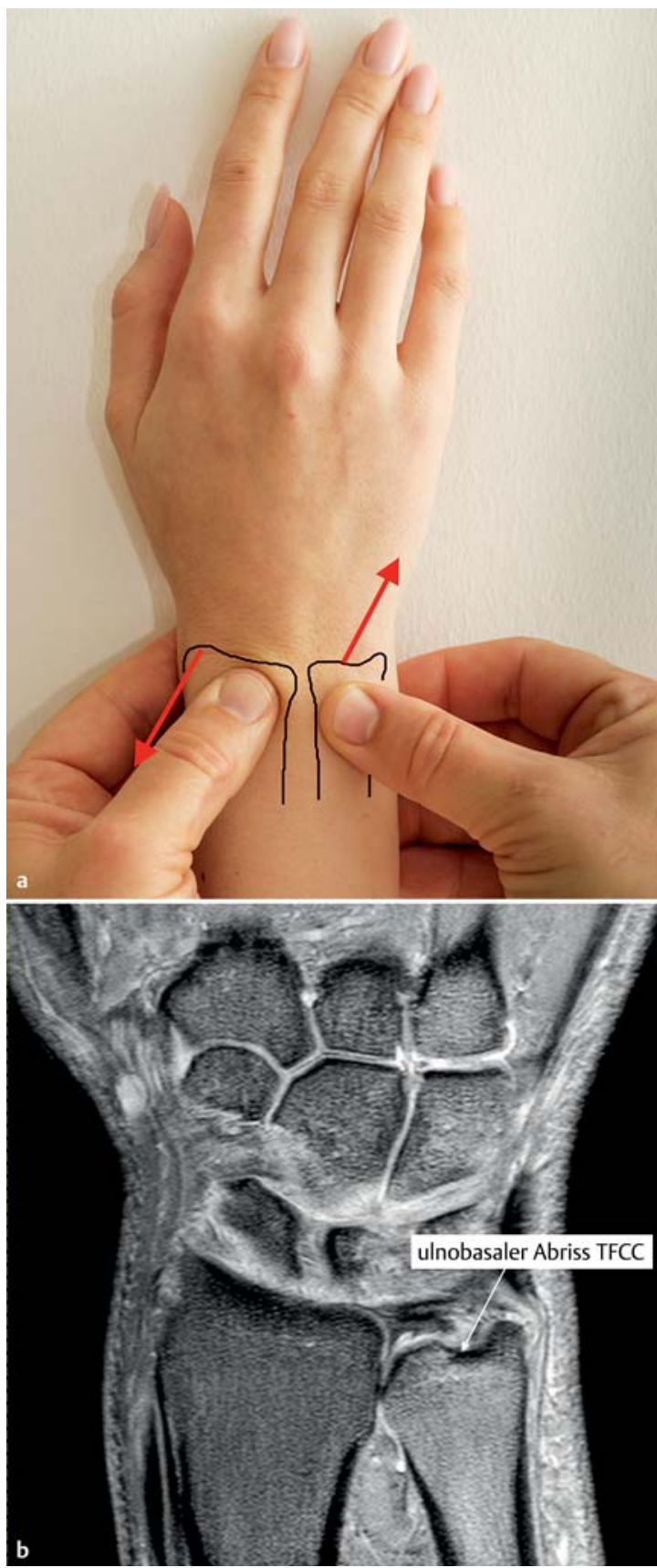

Abb. 7 a DRUG Instability Test. b MRT: ulnobasaler Abriss TFCC (koronal PD fettgesättigt). 

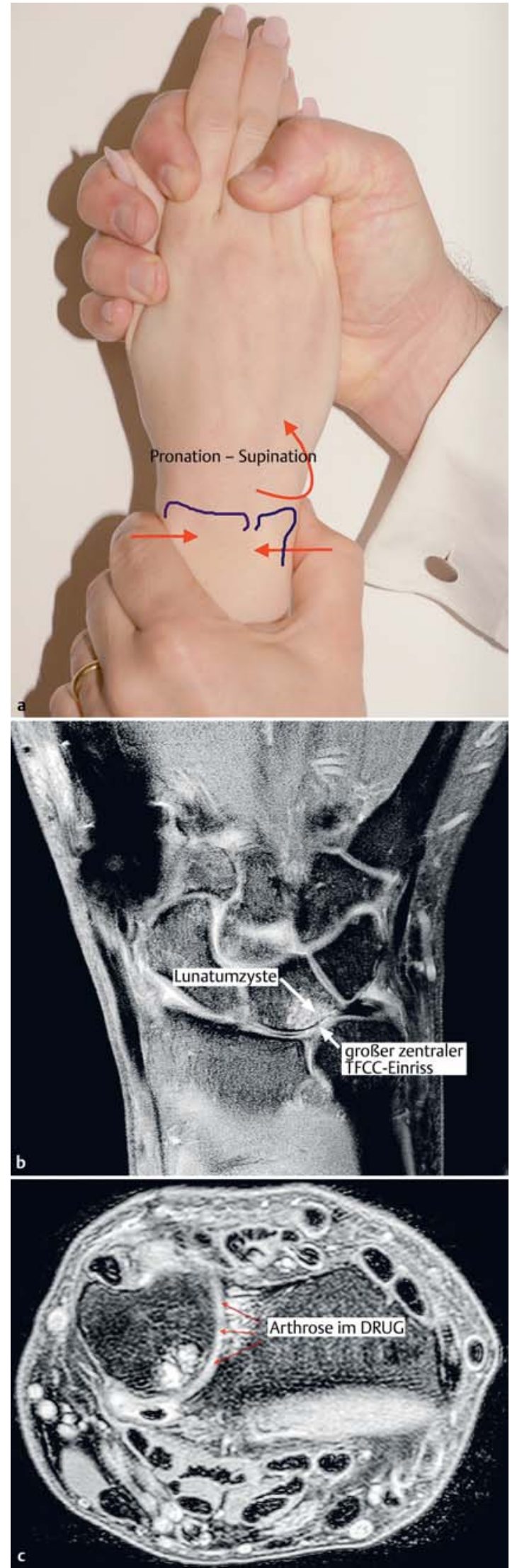

Abb. 8 a Radioulnar Compression Test. b MRT: Ulnolunar Impaction Syndrome (koronal, PD fettgesättigt). c MRT: Arthrose DRUG (axial, PD fettgesättigt).

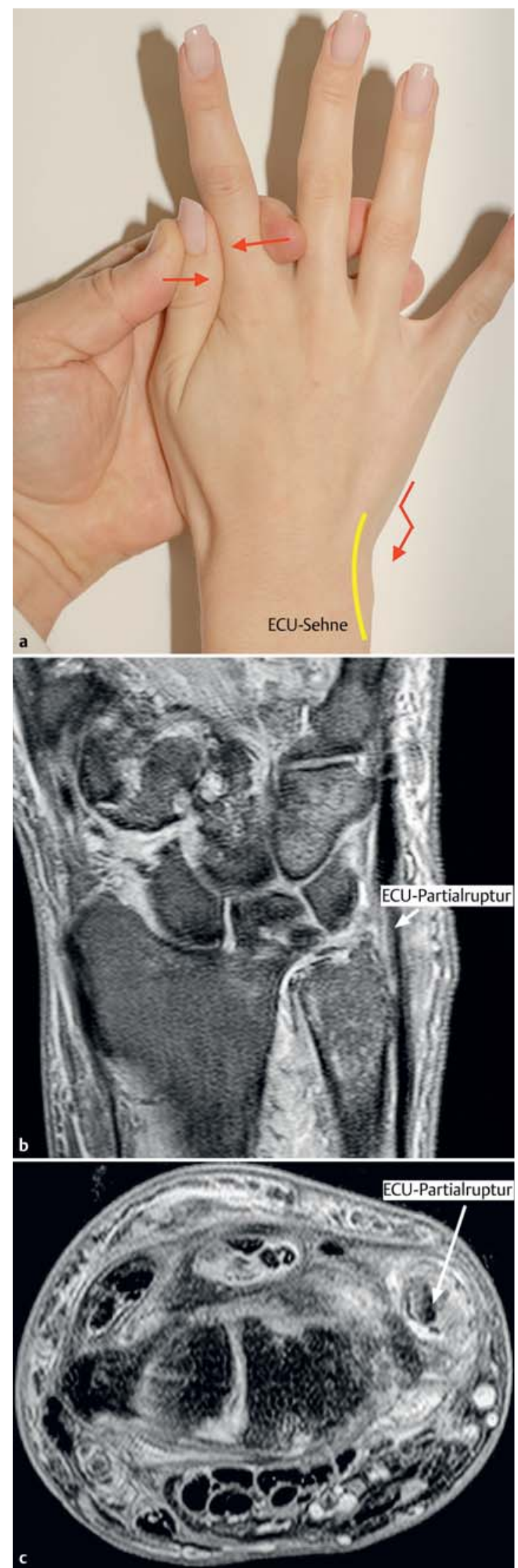

Abb. 9 a ECU Synergy Test. b MRT: Partialruptur ECU-Sehne (koronal, PD fettgesättigt). c MRT: Partialruptur ECU-Sehne (axial, PD fettgesättigt). 

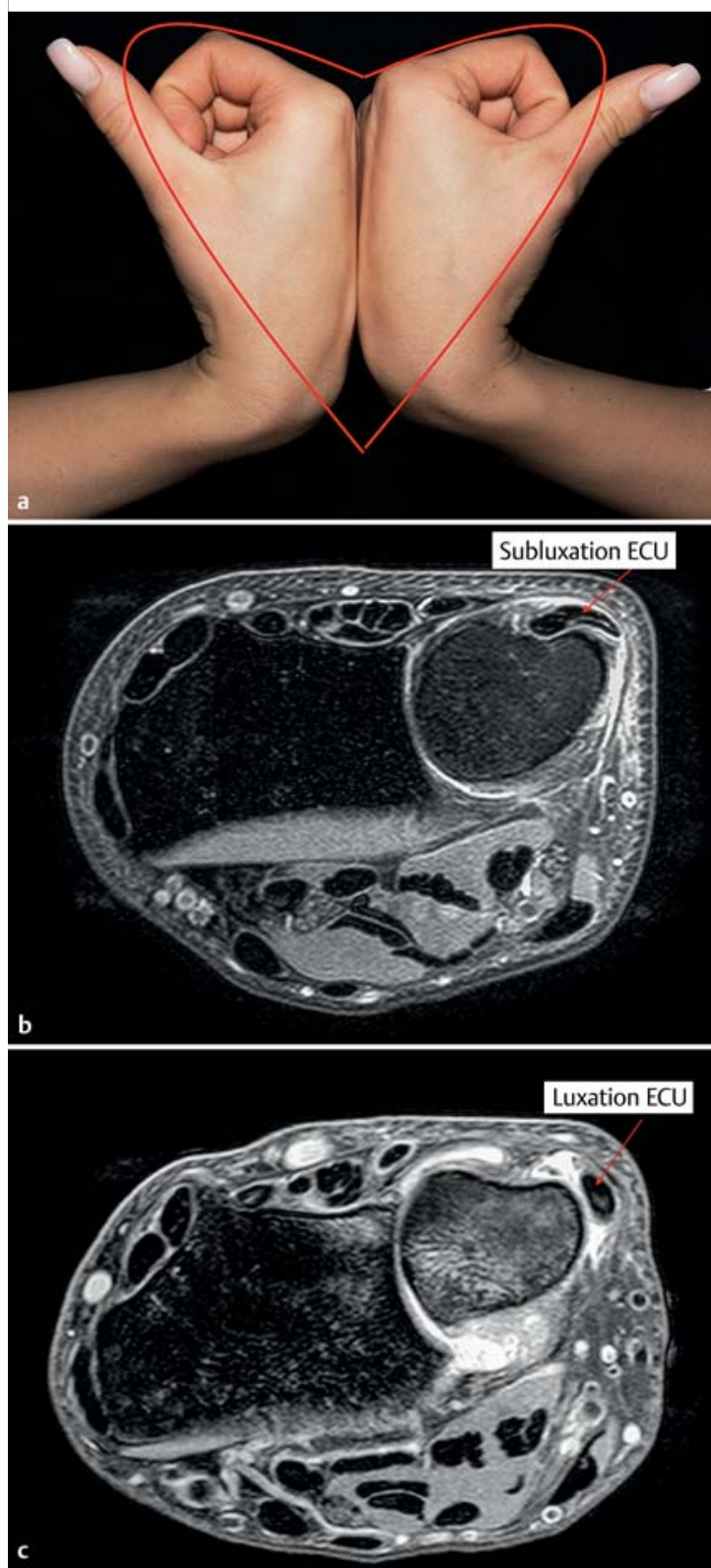

Abb. 10 a Heart-like Test. b MRT: Subluxation ECU-Sehne (axial PD fettgesättigt). c MRT: Luxation ECU-Sehne (axial PD fettgesättigt).

\section{Heart-like Test}

Der Patient wird gebeten, beide Handgelenke maximal zu beugen und die Handrücken gegeneinander zu pressen. Gleichzeitig drückt der ulnare Rand des Handgelenks gegen die Brust (Abb.10). Durch diesen Test (Garcia-Elias [16]) werden die 3 Bewegungen durchgeführt, die eine Dislokation der ECU-Sehne provozieren können: Flexion, Supination und Ulnarduktion gegen Widerstand. Die gegeneinander gepressten Hände imitieren die Gestalt eines Herzens. Nach Angabe des Autors ist der Test sehr sensibel und hat eine exzellente Spezifität.

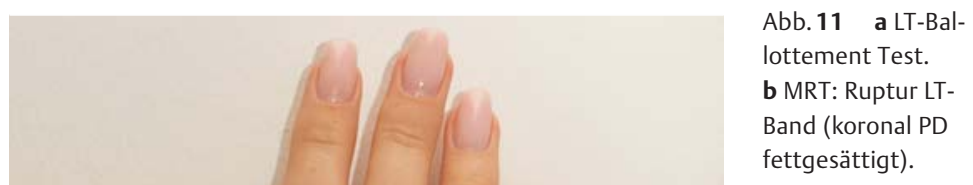

a

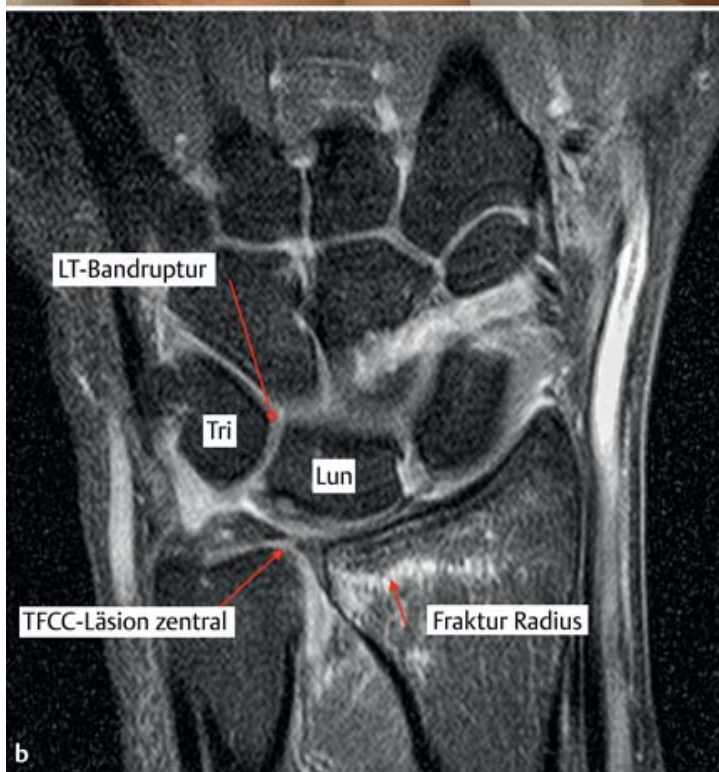

\section{Untersuchungstechnik: lunotriquetrales Gelenk}

\section{LT-Ballottement Test}

Von Reagan u. Mitarb. [17] 1984 beschrieben, kann mit diesem Test eine pathologische Hypermobilität im LTSpalt als Zeichen einer Verletzung des LT-Bandes dargestellt werden. 


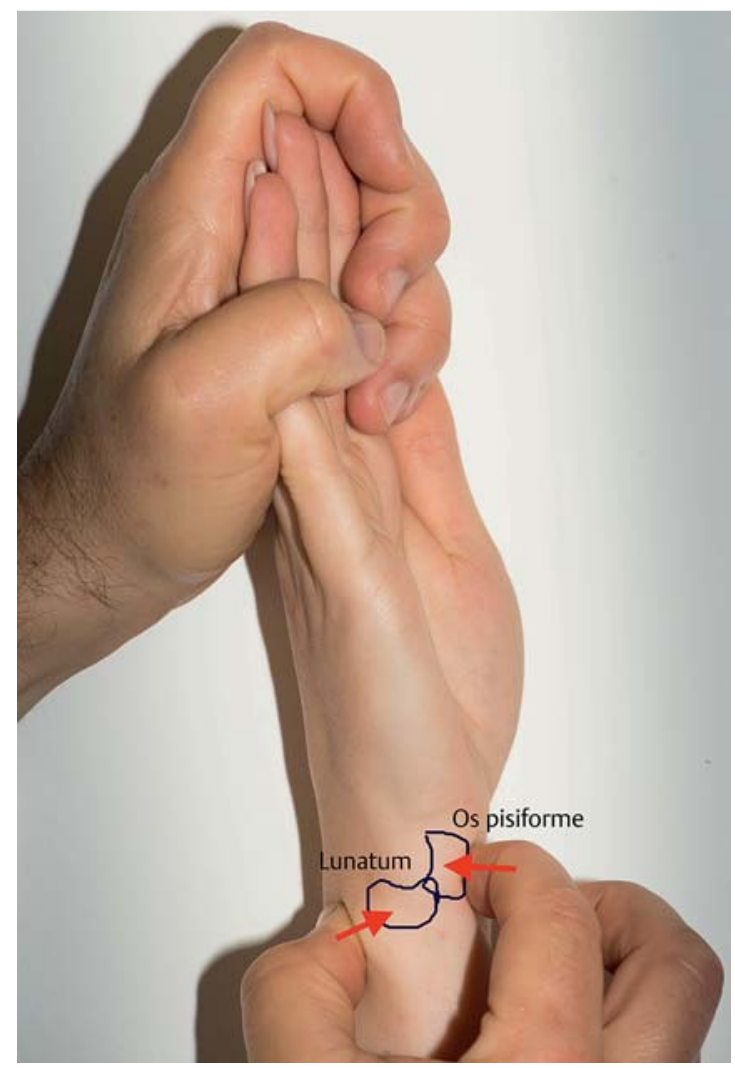

Abb. 12 Lunotriquetral Shear Test.

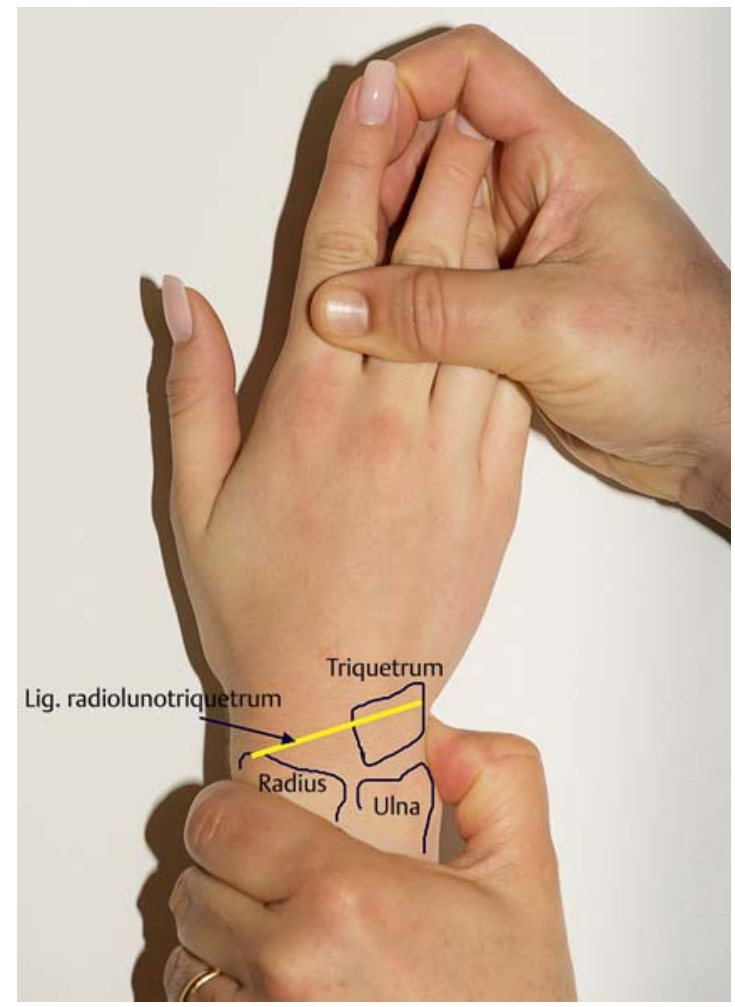

Abb. 13 Lunotriquetral Compression Test (Neutralstellung Handgelenk).
Die Hand des Patienten steht in Pronation, der Untersucher fixiert mit einer Hand das Lunatum und löst mit der anderen Hand am Triquetrum eine dorsopalmare Scherbewegung aus (Abb. 11).

\section{- Lunotriquetral Shear Test}

Dieser von Kleinman [18] propagierte Test zum Nachweis einer Instabilität im LT-Gelenk kann mit einer Hand durchgeführt werden: Das Handgelenk steht in Supination, gegenläufiger Druck wird von Daumen und Zeigefinger auf Lunatum und Pisiforme ausgeübt (Abb. 12).

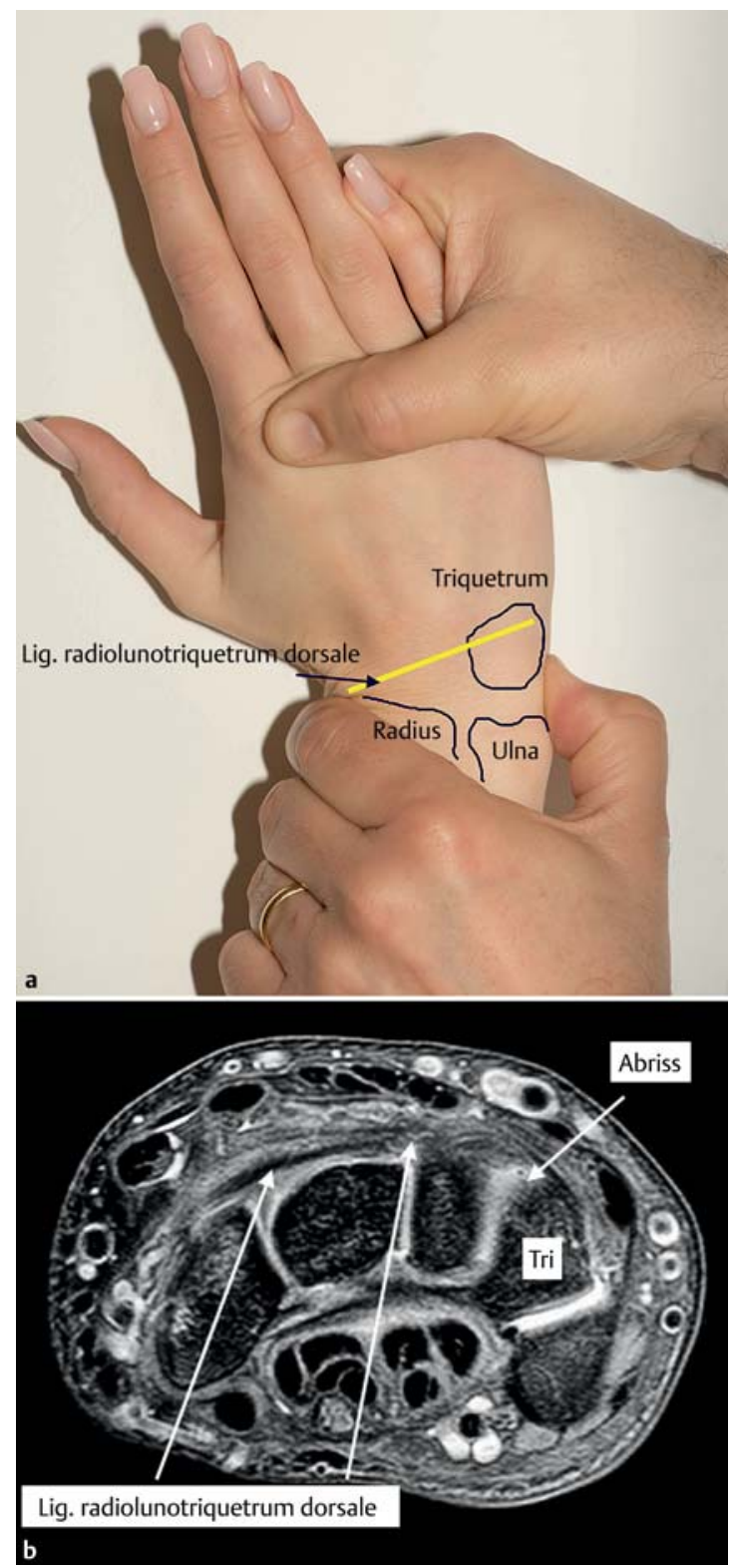

Abb. 14 a Lunotriquetral Compression Test (Radialduktion Handgelenk). b MRT: TILT-Syndrom (axial PD fettgesättigt). 


\section{Untersuchungstechnik: Triquetral Impingement Ligament Tear}

Watson [19] hat das TILT-Syndrom als eine Verletzung des „ulnar cuff“ beschrieben. Präzise MRT-Untersuchungen konnten zeigen, dass tatsächlich ein Ausriss des Lig. radiolunotriquetrum dorsale vorliegt. Dieser Ausriss kann knöchern oder bindegewebig erfolgen; der knöcherne Ausriss lässt sich meist leicht im seitlichen Röntgenbild erkennen. Spontanheilungen sind die Regel. In einigen Fällen löst der Bandabriss eine reaktive Synovitis zwischen Triquetrum und Hamatum aus. Eine mögliche Verletzung lässt sich mit dem im Folgenden genannten Test verifizieren.

\section{- Lunotriquetral Compression Test}

Nach Berger [9] entspricht dieser Test Linscheid's Lunotriquetral Shear Test. Das Handgelenk steht in Pronation, der Untersucher palpiert die ulnare Kante des Triquetrums mit dem Daumen und löst Druck aus. Anschließend wird das Handgelenk bei gleichbleibendem Druck nach radial duziert (Abb.13, Abb.14). Wird Schmerz provoziert, muss differenzialdiagnostisch neben dem TILT eine Pathologie im LT-Spalt berücksichtigt werden.

\section{Untersuchungstechnik: Os pisiforme}

\section{- Pisotriquetral Grind Test}

An der Palmarseite des Handgelenks liegt das Os pisiforme unmittelbar distal der Ulna. Bei adipösen oder ödematösen Händen lässt sich der Knochen durch leichte Flexion und Ulnarduktion des Handgelenks besser palpieren. Daumen und Zeigfinger umgreifen das Pisiforme und verschieben es nach medial und lateral unter beständigem Druck auf das Triquetrum (Abb. 15). Helal [20] hat gezeigt, dass Schmerz oder Krepitieren Hinweise auf eine pisotriquetrale Arthrose sind. Eine pathologische Subluxation des Knochens, die selten vorliegt, kann nur mit diesem Test festgestellt werden.

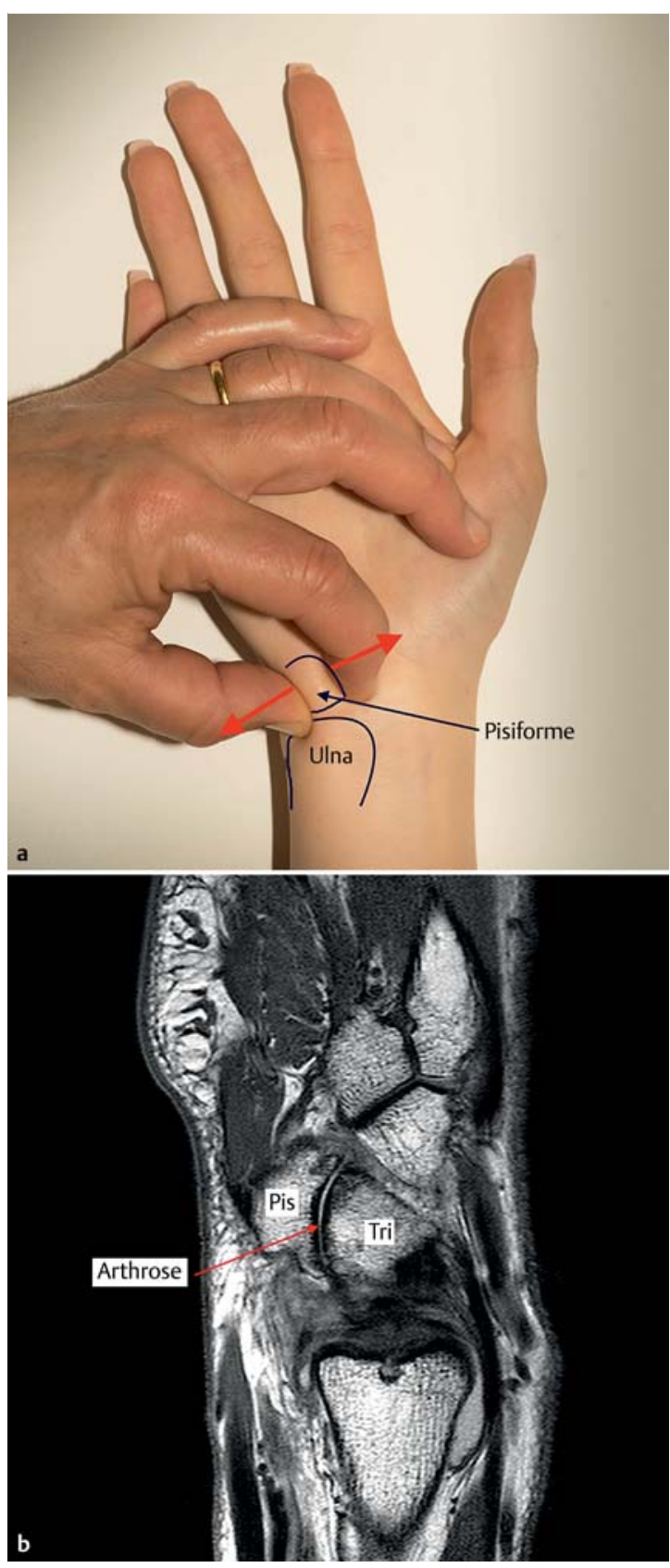

Abb.15 a Pisotriquetral Grind Test. b MRT: Arthrose pisotriquetral (sagittal PD fettgesättigt). 
Abb. 16 a Hook of the Hamate Pull Test. b MRT: Fraktur Hamulus ossis hamati (axial T1).
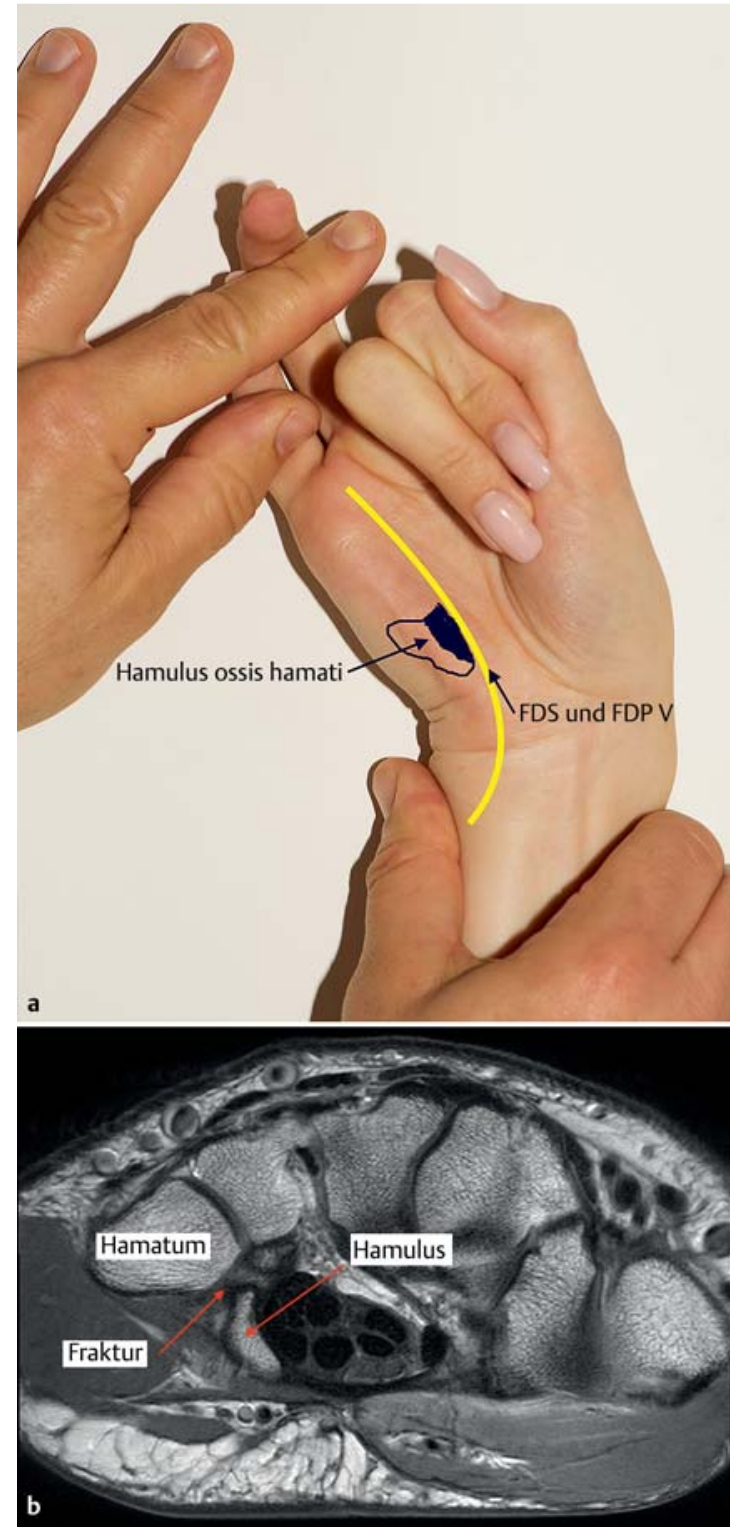

\section{Untersuchungstechnik: Hamulus ossis hamati}

\section{- Hook of the Hamate Pull Test}

Der Hamulus ossis hamati ist ähnlich wie das Tuberculum dorsale ein Hypomochlion: Hier werden die beiden Beugesehnen des Kleinfingers umgeleitet, um ein besseres Kraftmoment ausüben zu können. Dieser Test nach Wright u. Mitarb. [21] beruht auf der Erkenntnis, dass eine Kontraktion der genannten Sehnen eine ulnargerichtete Kraft auf den Hamulus auslöst. Das Handgelenk steht in Supination und Ulnarduktion. Der Untersucher hält Ring- und Kleinfinger fest und fordert den Patienten zur Flexion dieser Finger auf (Abb. 16). Die isometrische Kontraktion provoziert Schmerz bei Verletzungen des Hamulus (Fraktur, Pseudarthrose).

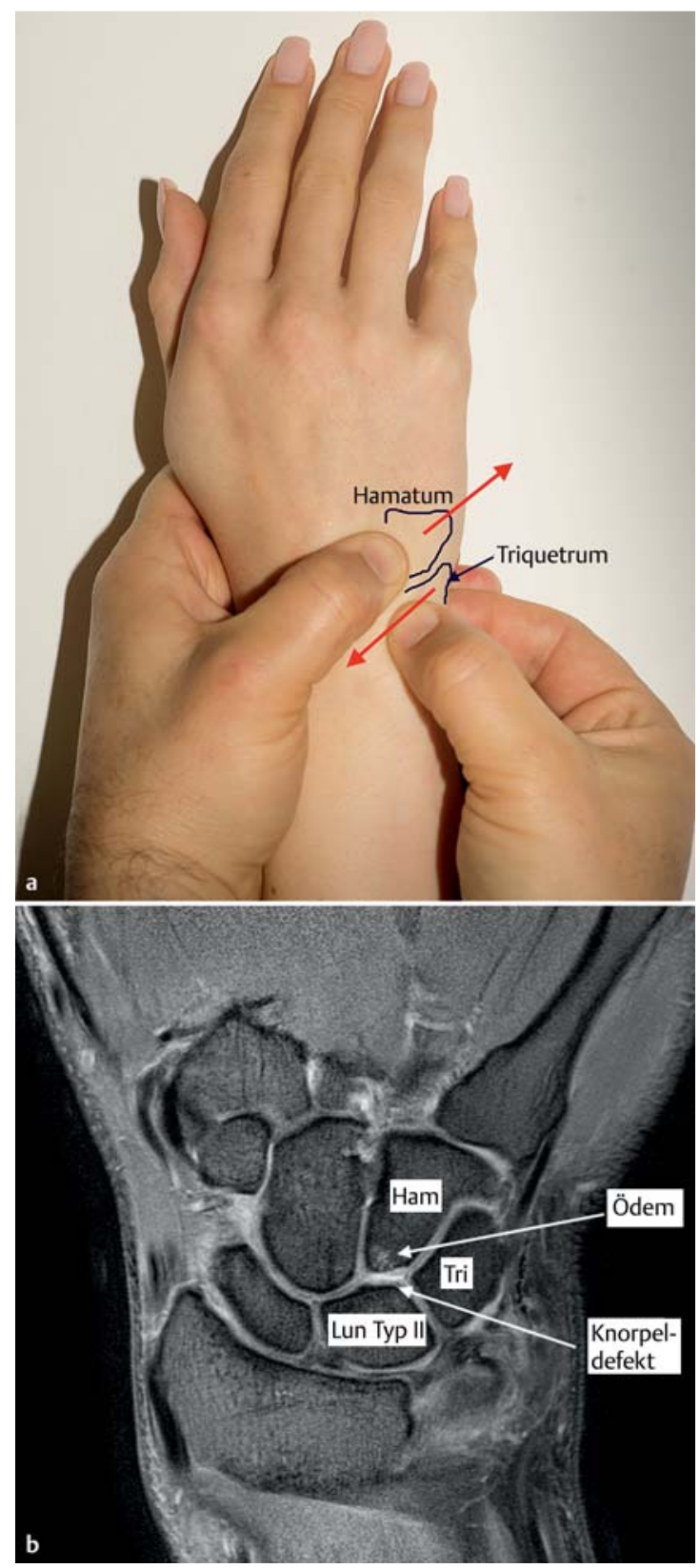

Abb. 17 a Triquetrohamate Shear Test. b MRT: Typ-II-Lunatum mit Knorpeldefekt Hamatum (koronal T2).

\section{Untersuchungstechnik: Triquetrum, Hamatum}

\section{- Triquetrohamate Shear Test}

Das Handgelenk steht in Pronation und leichter Ulnarduktion. Der Untersucher umfasst mit einer Hand das Hamatum, mit der anderen Hand das Triquetrum und löst eine Scherbewegung in dorsopalmarer Richtung aus (Abb. 17). Der Test ist laut Berger [9] hoch sensitiv und spezifisch bei Chondromalazie des proximalen Hamatumpols, ausgelöst durch die Formvariante eines 


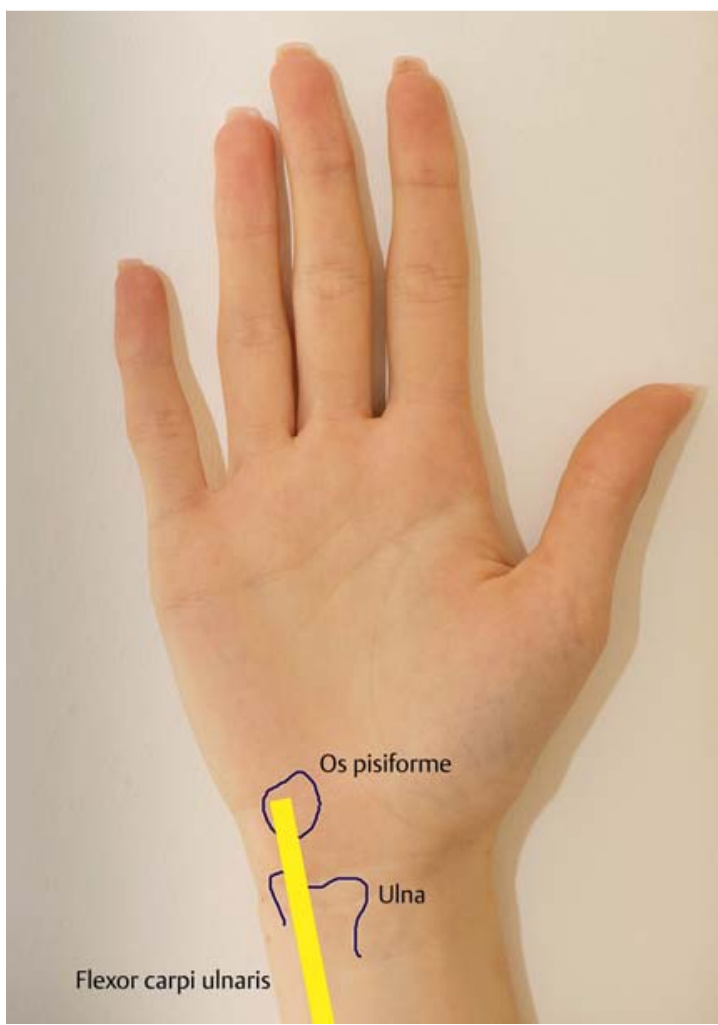

Abb. 18 FCU-Sehne.

Os lunatum Typ II. Auch Infraktionen der betroffenen Knochen („bone bruise“) können so entdeckt werden. Frakturen des Hamatum oder Triquetrum sind selten und können aufgrund der Schmerzhaftigkeit kaum palpiert werden.

\section{Untersuchungstechnik: Flexor-carpi- ulnaris-Sehne}

Ein spezifischer Test zur Untersuchung der FCU-Sehne existiert nicht. Dennoch darf sie nicht aus dem Untersuchungsprotokoll ausgeschlossen werden. In Supinationsstellung des Handgelenks lässt sich die Sehne meist einfach bei Flexion des Handgelenks palpieren (Abb. 18). Schmerzen im Verlauf der Sehne können ein Hinweis auf eine Partialruptur oder Synovialitis sein.

In Tab. 2 sind nochmals alle beschriebenen Tests aufgelistet und - da durch einzelne Test mehrere Pathologien aufgedeckt werden können - die entsprechenden möglichen Diagnosen aufgeführt.

\section{Tabelle 2}

Untersuchungstechnik und mögliche Pathologien ulnokarpal.

\begin{tabular}{|c|c|}
\hline Test & Pathologie \\
\hline Fovea Sign & $\begin{array}{l}\text { TFCC-Läsion ulnobasal, Verletzung Lig. ulnotri- } \\
\text { quetrum }\end{array}$ \\
\hline TFC Shear Test & TFCC-Läsion, Arthrose DRUG, Instabilität DRUG \\
\hline Ulnocarpal Stress Test & TFCC-Läsion, Ulna Impaction Syndrome \\
\hline Piano Key Test & Instabilität DRUG \\
\hline DRUG Instability Test & Instabilität DRUG \\
\hline Radioulnar Compression Test & Arthrose DRUG \\
\hline ECU Synergy Test & ECU-Tenosynovitis \\
\hline Heart-like Test & ECU-Instabilität (Subluxation, Luxation) \\
\hline LT-Ballottement Test & Verletzung oder Instabilität LT-Band \\
\hline Lunotriquetral Shear Test & Verletzung oder Instabilität LT-Band \\
\hline $\begin{array}{l}\text { Lunotriquetral } \\
\text { Compression Test }\end{array}$ & $\begin{array}{l}\text { TILT, LT-Arthrose, -Synovitis, -Instabilität, } \\
\text { Synchondrose }\end{array}$ \\
\hline Pisotriquetral Grind Test & $\begin{array}{l}\text { Arthrose pisotriquetral, mediale Subluxation } \\
\text { (selten!) }\end{array}$ \\
\hline Hook of the Hamate Pull Test & Fraktur oder Pseudarthrose Hamulus ossis hamati \\
\hline Triquetrohamate Shift Test & $\begin{array}{l}\text { Bone Bruise Triquetrum oder Hamatum, } \\
\text { TYP-II-Lunatum }\end{array}$ \\
\hline
\end{tabular}

Interessenkonflikt: Es liegt kein Interessenkonflikt vor.

\section{Danksagung}

Ich danke meinen radiologischen Kollegen Dr. Wolfgang Fischer und Dr. Peter Mundinger für die Durchführung der MRT-Untersuchungen. Ohne ihren unermüdlichen Einsatz und ihr stetes Streben nach Verbesserung der Bildgebung wäre exakte Diagnostik nicht möglich. Von ihnen und meinen Patienten habe ich unendlich viel gelernt. 


\section{Über den Autor}

\section{Ulrich Frank}

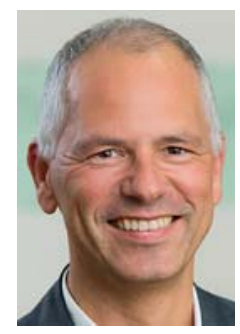

Dr. med. 1983-1990 Studium der Humanmedizin an der Ludwig-Maximilians-Universität München. September 1990 bis März 1992 AiP am Anatomischen Institut der Universität Bonn (Prof. Dr. med. H.-M. Schmidt). Approbation am 01.03.1992. Promotion am 26.03.1992. März 1992 bis Mai 1993 wissenschaftlicher Assistent am Anatomischen Institut der Universität Bonn (Prof. Dr. med. H.-M. Schmidt). Juni 1993 bis Mai 1996 wissenschaftlicher Assistent an der Klinik für Handchirurgie, Bad Neustadt/Saale (Prof. Dr. med. U. Lanz). Juni 1996 bis Juni 1998 wissenschaftlicher Assistent an der Orthopädischen Universitätsklinik im Oskar-Helene-Heim, Berlin (Univ.Prof. Dr. med. U. Weber). Juli 1998 bis September 2000 wissenschaftlicher Assistent an der Orthopädischen Klinik München-Harlaching (PD Dr. med. H. Mayer, Prof. Dr. med. R. Wetzel). Facharzt für Orthopädie am 20.09.2000. September 2000 bis September 2002 Oberarzt am Zentrum für Hand- und Rekonstruktive Mikrochirurgie (Dr. med. B. Lukas). Seit 1.10.2002 Leiter Handchirurgie der Hessingpark-Clinic Augsburg.

\section{Korrespondenzadresse}

Dr. Ulrich Frank

Leiter Handchirurgie

Hessingpark-Clinic

Hessingstraße 17

86199 Augsburg

ulrich.frank@hessingpark-clinic.de

\section{Literatur}

1 Shin AY, Deitch MA, Sachar K et al. Ulnar-sided wrist pain: diagnosis and treatment. Instr Course Lect 2005; 54: 115 128

2 Garcia-Elias M. Clinical examination of the ulnar-sided painful wrist. In: del Piñal F, Mathoulin C, Nakamura T, eds. Arthroscopic Management of Ulnar Pain. Berlin: Springer; 2012: 26

3 Watson HK, Weinzweig J. Physical examination of the Wrist. Hand Clin 1997; 13: 17- 34

4 Scharizer E. Klinische Untersuchung der Hand. Nigst H, Scharizer E, Hrsg. Untersuchung der Hand. Stuttgart: Hippokrates; 1991: 29-31

5 Shaaban H, Giakas G, Bolton M et al. The Distal Radioulnar Joint as a load-bearing mechanism - a biomechanical study. J Hand Surg 2004; 29A: 85-95

6 LaStayo PC, Weiss P. The GRIT: A quantitative measure of Ulnar Impaction Syndrome. J Hand Ther 2001; 14: 173-179

7 Tay SC, Tomita K, Berger RA. The "ulnar fovea sign" for defining ulnar wrist pain: an analysis of sensitivity and specificity. J Hand Surg 2007; 32A: 438-444

8 Nakamura T. Anatomy and biomechanics of the DRUJ. In: del Piñal F, Mathoulin C, Nakamura T, eds. Arthroscopic Management of Ulnar Pain. Berlin: Springer; 2012

9 Berger RA, Dobyns JH. Physical examination and provocative maneuvers of the wrist. In: Gilula LA, Yin Y, eds. Imaging of the wrist and hand. Philadelphia: WB Saunders; 1996

10 LaStayo P, Howell J. Clinical provocative tests used in evaluating wrist pain: a descriptive study. J Hand Ther 1995; 8: 10 14

11 Nakamura R, Horii E, Imaeda T. The ulnocarpal stress test in the diagnosis of ulnar-sided wrist pain. J Hand Surg 1997; 22B: $719-723$

12 Beckenbaugh RD. Accurate evaluation and management of the painful wrist following injury. Orthop Clin North Am 1984; 15: $289-306$

13 Morrissy RT, Nalebuff EA. Dislocation of the distal radioulnar joint: antomy and clues to prompt diagnosis. Clin Orthop Relat Res 1979; 144: 154-158

14 Cooney WP, Bishop AT, Linscheid RL. Physical examination of the wrist. In: Cooney WP, Linscheid RL, Dobyns JH, eds. The Wrist: Diagnosis and Operative Treatment. Maryland Heights, MO: Mosby; 1997

15 Ruland RT, Hogan CJ. The ECU Synergy Test: An aid to diagnose ECU tendonitis. J Hand Surg 2008; 33A: 1777 - 1782

16 Garcia-Elias M. Tendinopathies of the extensor carpi ulnaris. Handchir Mikrochir Plast Chir 2015; 47: 281 - 289

17 Reagan DS, Linscheid RL, Dobyns JH. Lunotriquetral sprains. J Hand Surg 1984; 9A: $502-551$

18 Kleinman WB. Stability of the distal radioulnar joint: biomechanics, pathophysiology, physical diagnosis, and restauration of function: what we have learned in 25 years. J Hand Surg 2007; 32A: $1086-1106$

19 Watson HK, Weinzweig J. Triquetral impingement ligament tear syndrome. J Hand Surg 1999; 24B: 350-358

20 Helal B. Racquet's players pisiform. Hand 1978; 10: 87-90

21 Wright TW, Moser MW, Sahajpal DT. Hook of the hamate pull test. J Hand Surg 2010; 35A: 1887 - 1889 


\section{CME-Fragen}

\section{CME•thieme.de}

\section{CME-Teilnahme}

\Viel Erfolg bei Ihrer CME-Teilnahme unter http://cme.thieme.de

Bitte informieren Sie sich vorab online über die Gültigkeitsdauer.

- Sollten Sie Fragen zur Online-Teilnahme haben, unter http://cme.thieme.de/hilfe

finden Sie eine ausführliche Anleitung.

\section{1}

Welche Aussage ist falsch? Welche Strukturen gehören zum TFCC?

Welche Aussage ist richtig?
A ECU-Sehnenscheide
B Discus triangularis
C Lig. radiolunotriquetrum dorsale
D Lig. ulnolunatum
E Meniscus homologue

A Der Ulnakopf ist nicht palpabel.

B Der Processus styloideus ulnae liegt bei Pronation des Handgelenks dorsal.

C $25 \%$ der Schmerzen ulnokarpal sind intrakapsulär.

D Das Os pisiforme liegt radial des Hamulus ossis hamati.

E $25 \%$ der Schmerzen ulnokarpal sind extrakapsulär.

3

Welche Aussage ist falsch?
A Die Fovea ulnae kann von ulnar leicht palpiert werden.
B Die Fovea ulnae liegt dorsal des Extensor carpi radialis.
C Die Fovea ulnae wird auch durch das Triquetrum begrenzt.
D Der R. dorsalis n. ulnaris kreuzt die „foveal region“.
E Schmerz in der Fovea kann ein Hinweis auf eine ulnobasale Ruptur des TFCC sein.

4

Welche Aussage ist richtig?

A Die ECU-Sehne ist bei Rotationsverletzungen des Handgelenks nicht gefährdet.
B Der Hamulus ossis hamati verheilt nach Fraktur immer.

C Axiale Belastung des TFCC nach Verletzungen kann in der Regel schmerzfrei durchgeführt werden.

D Das „dimple sign“ ist ein Hinweis auf eine Instabilität im DRUG.

E Der Hook of the Hamate Pull Test dient als Nachweis einer Beugesehnenverletzung.

5

Welche Aussage ist falsch?
A LT-Band-Verletzungen können durch den LT-Ballottement Test aufgespürt werden.

B Der LT Shear Test wird mit einer Hand durchgeführt.

C Der positive Piano Key Test ist Hinweis auf eine LT-Band-Verletzung.

D Der ulnobasale Abriss des TFCC kann zu einer Instabilität im DRUG führen.

E Eine ECU-Synovitis wird häufig als TFCC-Läsion fehldiagnostiziert. 


\section{CME-Fragen}

Untersuchung und MR-Morphologie des ulnokarpalen Handgelenkschmerzes

\section{6}

Welche Aussage ist richtig? Eine MRT-Untersuchung ist sinnvoll ...
A bei jedem Patienten mit Kontrastmittel.
B bei fehlverheilter Radiusfraktur.
C bei positivem Piano Key Test.
D ohne klinische Untersuchung bei jedem Handgelenkschmerz.
E bei Platzangst des Patienten.

\section{7}

Welche Aussage ist falsch?
A Der Radioulnar Compression Test kann Hinweise auf eine Arthrose im DRUG geben.
B Die FCU-Sehne ist selten verletzt.
C Triquetrumfrakturen sind selten.
D Eine Verletzung des SL-Bands ist häufiger als eine LT-Bandverletzung.
E Der ECU Synergy Test ist ein wertvoller Test zur Beurteilung einer Sehnenluxation.

\section{8}

Welche Aussage ist richtig? Das TILT-Syndrom ...
A ist eine LT-Band-Verletzung.
B ist ein Abriss des Lig. radiolunotriquetrum dorsale.
C ist eine Verletzung des Lig. ulnotriquetrum.
D zeigt einen positiven Radioulnar Compression Test.
E kann im MRT nicht dargestellt werden.

9

Welche Aussage ist falsch?
A Ulnokarpaler Schmerz ist bei Frauen häufiger als bei Männern.
B Untersuchung der Beweglichkeit muss an beiden Handgelenken durchgeführt werden.
C Die Dynamometeruntersuchung gibt wertvolle Hinweise auf schmerzbedingte Kraftminderung.
D Rheumapatienten zeigen häufig eine ECU-Synovitis.
E Ulnokarpaler Schmerz kann auch neurogen bedingt sein.

10
A Der Heart-like Test wird häufig von Partnerschaftsvermittlungen eingesetzt.
B Bei ulnokarpalem Schmerz gilt: „Ein gutes CT ist besser als ein schlechtes MRT.“
C Ein ulnobasaler Abriss des TFCC lässt sich am einfachsten arthroskopisch darstellen.
D Ein Os lunatum Typ II kann eine Prädisposition für eine Chondromalazie am Hamatum sein.
E Der Pisotriquetral Grind Test gibt Hinweis auf eine TFCC-Läsion. 